Please do not remove this page

RMIT

UNIVERSITY

\title{
GPS signal acquisition and tracking using software GPS receiver
}

Wu, Falin; Zhang, Kefei; Yasuda, Akio

https://researchrepository.rmit.edu.au/esploro/outputs/9921858623501341/filesAndLinks?institution=61RMIT_INST\&index=null

Wu, F., Zhang, K., \& Yasuda, A. (2005). GPS signal acquisition and tracking using software GPS receiver. Proceedings of the Eighth International Symposium on Signal Processing and Its Applications, 843-846. https://researchrepository.rmit.edu.au/discovery/fulldisplay/alma9921858623501341/61RMIT_INST:Resea rchRepository

Document Version: Published Version

Repository homepage: https://researchrepository.rmit.edu.au

(C2005 IEEE. Personal use of this material is permitted. However, permission to reprint/republish this material for advertising or promotional purposes or for creating new collective works for resale or redistribution to servers or lists, or to reuse any copyrighted component of this work in other works must be obtained from the IEEE.

Downloaded On 2023/04/26 14:47:36 +1000 


\section{GPS SIGNAL ACQUISITION AND TRACKING USING SOFTWARE GPS RECEIVER}

\author{
Falin $\mathrm{Wu}^{*}$ and Kefei Zhang \\ The RMIT University \\ Melbourne, Australia \\ *falin.wu@rmit.edu.au
}

\author{
Akio Yasuda \\ Tokyo University of Marine \\ Science and Technology \\ Tokyo, Japan
}

\begin{abstract}
In this paper we investigate GPS signal acquisition and tracking using software GPS receiver. The software GPS receiver consists of a RF front-end, an ADC, and a software GPS program that runs on PC. The RF front-end down-converts the signal from RF to IF, and the ADC samples the IF signal. All the other processing including signal acquisition, tracking, data decoding, and solving position are all implemented in software using signal processing techniques. The local C/A code and carrier replica signals are pre-generated, stored in memory, and used repetitively during signal acquisition and tracking. The concepts of signal processing using software GPS receiver can be applied to the next generation Global Navigation Satellite System and Space Based Augmentation System receivers design.
\end{abstract}

\section{INTRODUCTION}

The modernization of US Global Positioning System (GPS), the revival of Russian GLONASS and the advent of European Galileo System will bring the next generation Global Navigation Satellite System (GNSS). To augment GPS for aviation users the US, Europe and Japan have deployed the Wide Area Augmentation System (WAAS), the European Geostationary Navigation Overlay System (EGNOS) and MTSAT Satellite Augmentation System (MSAS), respectively. Each of these Space Based Augmentation System (SBAS) services is free and interoperable, providing coverage for most of North America, most of Europe and Japan, respectively. At the same time Japan launches a new regional SBAS plan, Japanese Quasi-Zenith Satellite System (QZSS), a constellation of three to seven satellite will broadcast GPS signals from orbits optimized for East Asia regions. Although GNSS and SBAS will provide users more navigation satellite signals, it is a challenge for users to design the next generation GNSS receivers.

Currently GPS receivers consist of a Radio Frequency (RF) front-end, an Applications Specific Integrated Circuit (AISC) for signal processing, and a CPU core for higher level functions. Firmware can be loaded into the CPU to change the performance parameters of the receiver. However, design flexibility is very constrained by an AISC that is hardwired with predefined tracking channels, correlator and control loop characteristics. So a software GNSS receiver is the best solution for next generation GNSS re- ceiver design. Software GNSS receivers are those that implement signal acquisition and tracking process not in hardware, but in software $[1,2]$. Each of GNSS, although different, operates in the required underlying signal processing. As such, the software GPS receiver architecture will be used through this paper as an example but the concepts can be applied to the design of any software GNSS receivers.

In this paper GPS signal acquisition and tracking using software GPS receiver are investigated. A prototype software GPS receiver is developed. The receiver is based on personal computer (PC) and samples GPS signal at intermediate frequency (IF). First, the structure of software GPS receiver and data collection hardware is presented. Second, the GPS Coarse/Acquisition (C/A) code acquisition is described. Then, the GPS signal tracking is discussed. Finally, some useful conclusions are given.

\section{SOFTWARE GPS RECEIVER}

Software GPS receiver uses an analog-to-digital converter (ADC) to change the input GPS signal into digital data at the earliest possible stage in the receiver. In other words, the input signal is digitized as close to the antenna as possible [2]. Fig. 1 illustrates a general structure of software GPS receiver [3, 4]. The signals transmitted from the GPS satellites are received from an antenna. Through a RF front-end, the input signal is amplified to a proper amplitude and the frequency is converted to a desired frequency. An ADC is used to digitize the input signal. The antenna, RF front-end, and ADC are the hardware used in the software GPS receiver. After the signal is digitized, a software is used to process it. Acquisition means to find the signal of a certain satellite. The tracking program is used to find the phase transition of the navigation data. From the navigation data phase transition, the sub-frames and navigation data can be obtained. Ephemeris data and pseudoranges can be obtained from the navigation data. The ephemeris data are used to obtain the satellite positions. Finally, the user position, velocity, and time can be calculated [2]. In this paper the GPS C/A code signals are down-converted from $1575.42 \mathrm{MHz}$ to $15.42 \mathrm{MHz}$ and sampled at $20 \mathrm{MHz}$ using a GPS SIGNAL TAP of Accord Software and Systems Private Company [5]. Therefore, the center of the digitized signal is at $4.78 \mathrm{MHz}$. The signal samples can be processed in real-time and/or stored on 


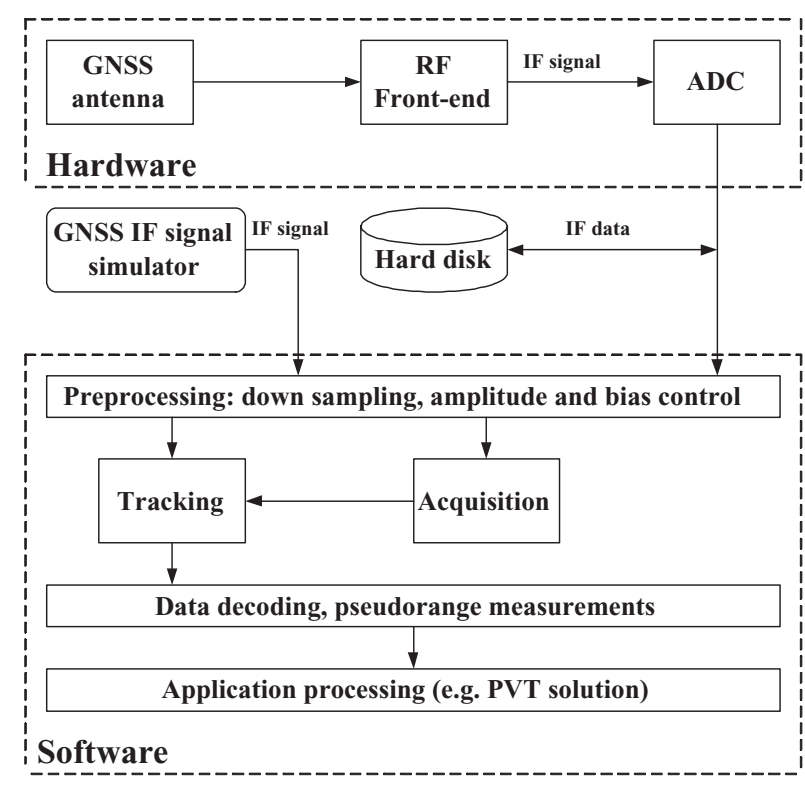

Fig. 1. Structure of a general GPS receiver

the hard disk for post processing.

\section{GPS C/A CODE ACQUISITION}

The local C/A code and carrier replica signals are pregenerated, stored in memory, and used repetitively during signal acquisition and tracking.

\subsection{GPS C/A Code Properties and Generation}

The signal structure of GPS may be modified in the future. However, at present, GPS satellites transmit two primary L-band signals, termed L1 and L2, having carrier frequencies of $1575.42 \mathrm{MHz}$ and $1227.6 \mathrm{MHz}$, respectively. The $\mathrm{L} 1$ frequency contains the $\mathrm{C} / \mathrm{A}$ and $\mathrm{P}(\mathrm{Y})$ signals, while the $\mathrm{L} 2$ frequency contains only the $\mathrm{P}(\mathrm{Y})$ signal. The $\mathrm{C} / \mathrm{A}$ and $\mathrm{P}(\mathrm{Y})$ signals in $\mathrm{L} 1$ frequency are in quadrant phase of each other and they can be written as [2]

$$
\begin{aligned}
S_{L 1}= & A_{P} P(t) D(t) \cos \left(2 \pi f_{1} t+\phi\right) \\
& +A_{C} C(t) D(t) \sin \left(2 \pi f_{1} t+\phi\right),
\end{aligned}
$$

where $S_{L 1}$ is the signal at L1 frequency, $A_{P}$ is the amplitude of the P code, $P(t)= \pm 1$ represents the phase of the P code, $D(t)= \pm 1$ represents the data code, $f_{1}$ is the $\mathrm{L} 1$ frequency, $\phi$ is the initial phase, $A_{C}$ is the amplitude of the C/A code, $C(t)= \pm 1$ represents the phase of the C/A code.

The C/A code is a bi-phase modulated signal with a chip rate of 1.023 MHz. Therefore, the null-to-null bandwidth of the main lobe of the spectrum is $2.046 \mathrm{MHz}$. Each chip is about $977.5 \mathrm{~ns}(1 / 1.023 \mathrm{MHz})$ long. The transmitting bandwidth of the GPS satellite in the L1 frequency is approximately $20 \mathrm{MHz}$ to accommodate the $\mathrm{P}$ code signal; therefore, the C/A code transmitted contains the main lobe and several side lobes. The total code period contains 1,023 chips. With a chip rate of $1.023 \mathrm{MHz}$,

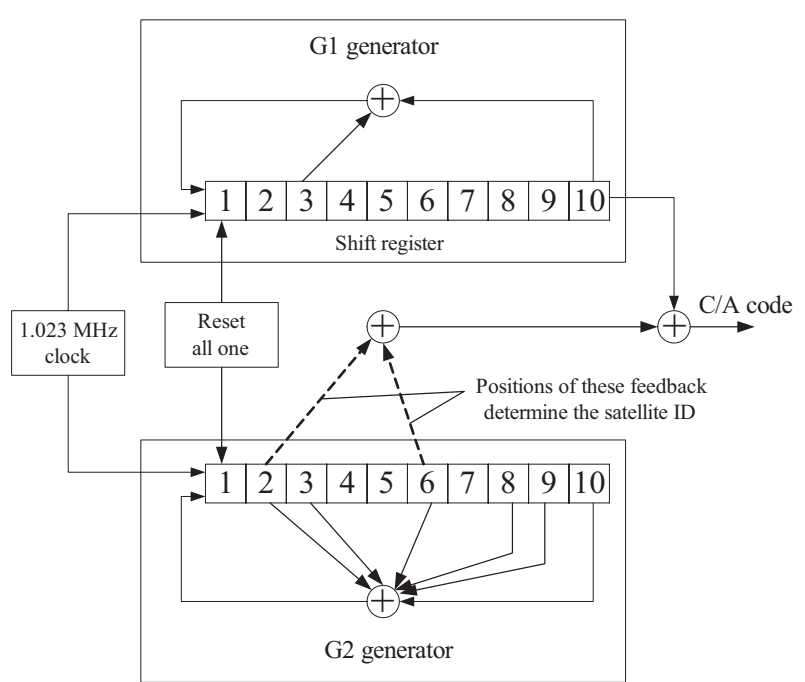

Fig. 2. C/A code generator

1,023 chips last $1 \mathrm{~ms}$; therefore, the C/A code is $1 \mathrm{~ms}$ long. The code repeats itself every millisecond.

In order to find the beginning of a C/A code in the received signal only a very limited data record is needed such as $1 \mathrm{~ms}$. If there is no Doppler effect on the received signal, then one millisecond of data contains all the 1,023 chips. A guaranteed way to receive the first three subframes is to take 30 seconds (or one page) of data. Thus, one can take a minimum of 30 seconds of data and calculate the user position [2].

The GPS C/A signals belong to the family of PseudoRandom Noise (PRN) codes known as the Gold codes [2]. Different C/A codes are used for different satellites. The signals are generated from the product of two 1,023-bit PRN sequences $G 1$ and $G 2$. Fig. 2 shows the C/A code generator. One of the most important properties of the C/A codes is their correlation. High autocorrelation peak and low cross-correlation peaks can provide a wide dynamic range for signal acquisition. The maximum of the autocorrelation peak is 1023 , which equals the C/A code length. The rest of the autocorrelation has three values 63, -1 , and -65 . The cross-correlation also has three values $63,-1$, and -65 [2].

\subsection{GPS C/A Code Acquisition}

The acquisition algorithm is used to determine whether a certain satellite is in the input signal, and if so determine its code phase and carrier frequency. The fast Fourier transform (FFT) approach will be used to acquire GPS C/A code.

The FFT approach performs circular convolution in the frequency domain. A noncoherent correlator in frequency domain can be adapted to the acquisition of GPS signals as shown in Fig. 3 [6]. The discrete Fourier transform (DFT) and its inverse is used to calculate the corre- 


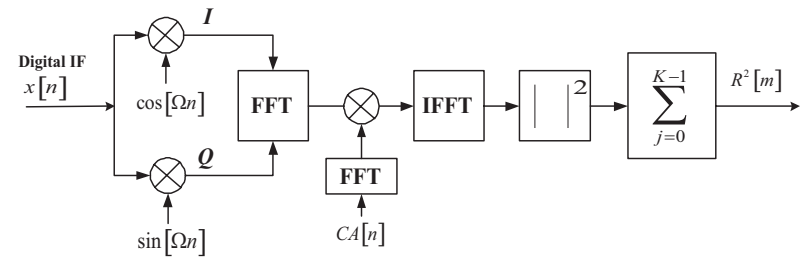

Fig. 3. Non coherent correlator in frequency domain

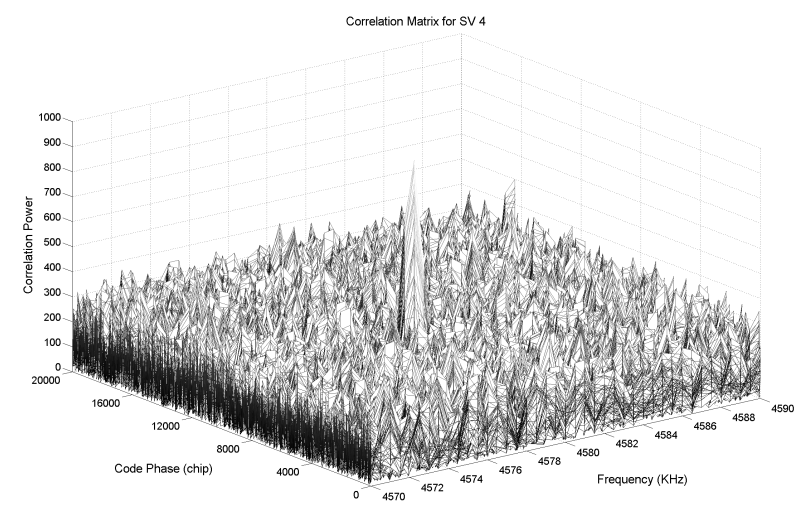

Fig. 4. Acquisition result using FFT approach (Satellite SV4)

lation value, $R[6]$,

$$
\begin{aligned}
R[m] & =\underbrace{x[n] \otimes C A[-n]}_{\text {Circular convolution }} \\
& =F^{-1}\left(F(x[n]) \cdot F(C A[n+m])^{*}\right),
\end{aligned}
$$

where $F, F^{-1}$ and ${ }^{*}$ denote FFT, inverse FFT and the complex conjugate, respectively. $n$ represents the $n^{\text {th }}$ sample and $m$ represents the number of samples that the replicated C/A code is phase shifted. First the input digitized IF signal, $x[n]$, is mixed to base band. After the carrier removal, the in-phase $(I)$ and quadrature-phase $(Q)$ components are used as the real and imaginary inputs when calculating the DFT. The result is multiplied by the complex conjugate of DFT of the C/A code, $C A[n+m]$. The circular convolution is obtained by taking the magnitude of the inverse DFT. The fast Fourier transform algorithm is used to implement the DFT and inverse DFT, hence the acquisition method may be called FFT approach.

Fig. 4 illustrates the result of acquisition using FFT approach. Because the Doppler frequency range that needs to be searched is $\pm 10 \mathrm{KHz}$, and the center of the signals is at $4.78 \mathrm{MHz}$, see Sec. 2. It is important to determine the frequency steps needed to cover range from $4.57 \mathrm{MHz}$ to $4.59 \mathrm{MHz}$. After the circular correlation, the highest component occurs at $4.578 \mathrm{MHz}$ and the beginning of the C/A code is at 7547 .

\section{GPS SIGNAL TRACKING}

Once a signal is acquired, the signal must be tracked in order to obtain the navigation data. The tracking program uses two parameters obtained from the acquisition

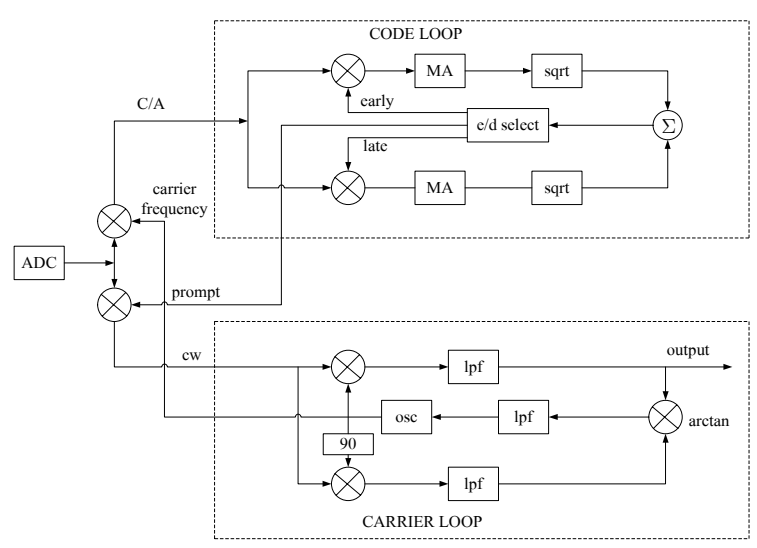

Fig. 5. Code and carrier tracking loops

process: the beginning of the C/A code period and the carrier frequency of the input signal to start the processing. Two loops are needed to track one GPS signal. One loop is often referred to as the code loop, which tracks the C/A code. The other one is the phase (or frequency) locked loop, which tracks the carrier frequency of the down-converted input signal. These two loops must be coupled together as shown in Fig. 5 [2].

The code loop uses three locally generated C/A codes to track the C/A code of the input signal. Three locally generated codes are usually used: a prompt, an early and a late code. The prompt code is applied to the digitized input signal and strips the C/A code from the input signal. The output will be a continuous wave (cw) signal with phase transition caused only by the navigation data, which is applied to the input of the carrier loop.

The output from the carrier loop is a cw with the carrier frequency of the input signal. This signal is used to strip the carrier from the input digitized signal. The output is a signal with only a C/A code and no carrier frequency, which is applied to the input of the code loop.

\subsection{Code Tracking}

The code loop is known as a delay lock loop (DLL) [7]. The prompt code is intended to match the C/A code in the input signal. The correlation outputs from three local codes can be used to accurately determine the beginning of the C/A code in the input signal. This information is used to adjust the initial phase of the locally generated prompt code to better match the code phase of the input signal. The discriminator algorithm is to give a calculated number on the code phase error. The discriminator output signal, $\varepsilon$, is calculated by

$$
\varepsilon=\frac{y_{E}}{y_{L}}
$$

where $y_{E}$ and $y_{L}$ are the correlation powers of the early code and the late code, respectively. If $\varepsilon=1$, the prompt code is perfectly aligned with the C/A code in the input signal; if $\varepsilon>1.1$, the local codes should be shifted to the right; if $\varepsilon<0.9$, the local codes should be shifted to the left [6]. 


\subsection{Carrier Tracking}

The phase locked loop is used to track the input IF signal, which is obtained from the acquisition program. The locally generated reference IF signal for the tracking program should be closer to the input IF signal.

A second-order loop can track the rate of change in Doppler and a third-order loop can be used to track constant acceleration [2]. For a software GPS receiver, the phase and frequency of the reference signal are adjusted every $\mathrm{ms}$ or $10 \mathrm{~ms}$. This operation may put the IF of the locally generated signal very close to the IF of the input signal, but may not be exactly equal to it. In this paper a second order PLL is used as the filter to track the input IF signal. The filter function is

$$
F(z)=\frac{\left(C_{1}+C_{2}\right)-C_{1} z^{-1}}{1-z^{-1}},
$$

where $C_{1}$ and $C_{2}$ are coefficients,

$$
\begin{aligned}
C_{1} & =\frac{1}{k_{0} k_{1}} \frac{8 \varsigma \omega_{n} t_{s}}{4+4 \varsigma \omega_{n} t_{s}+\left(\omega_{n} t_{s}\right)^{2}}, \\
C_{2} & =\frac{1}{k_{0} k_{1}} \frac{4\left(\omega_{n} t_{s}\right)^{2}}{4+4 \varsigma \omega_{n} t_{s}+\left(\omega_{n} t_{s}\right)^{2}},
\end{aligned}
$$

where $\omega_{n}$ is a natural frequency,

$$
\omega_{n}=\frac{2 \beta}{\zeta+\frac{1}{4 \zeta}},
$$

$\zeta$ is the damping ratio, $\beta$ is the bandwidth, $t_{s}$ is the sampling interval. The amplifier $k_{0}$ represents the gain of the phase comparator and the low-pass filter limits the noise in the loop, and $k_{1}$ is the gain of the voltage-controlled oscillator [2].

The results of GPS signal tracking using the tracking method proposed in this paper are illustrated in Fig. 6, which plots the amplitude of the output signal. The data from the satellite is taken from the prompt I-component before it is fed into the arctangent discriminator, see Fig. 5. Each data point is obtained from five milliseconds of digitized data. The amplitude changes with time; this is the transit effect of the tracking loop. Finally, the amplitude reaches a steady state.

\section{CONCLUSION}

In this paper we have investigated GPS signal acquisition and tracking using software GPS receiver. The FFT acquisition approach and the code and carrier tracking methods have been studied. A prototype software GPS receiver has been developed. The receiver is based on a PC and samples GPS signals at IF. The concepts of signal processing using software GPS receiver can be applied to any software GNSS receiver design. We are developing a genuine software GNSS receiver to acquire and track the signals of modernized GPS, Galileo, and QZSS.

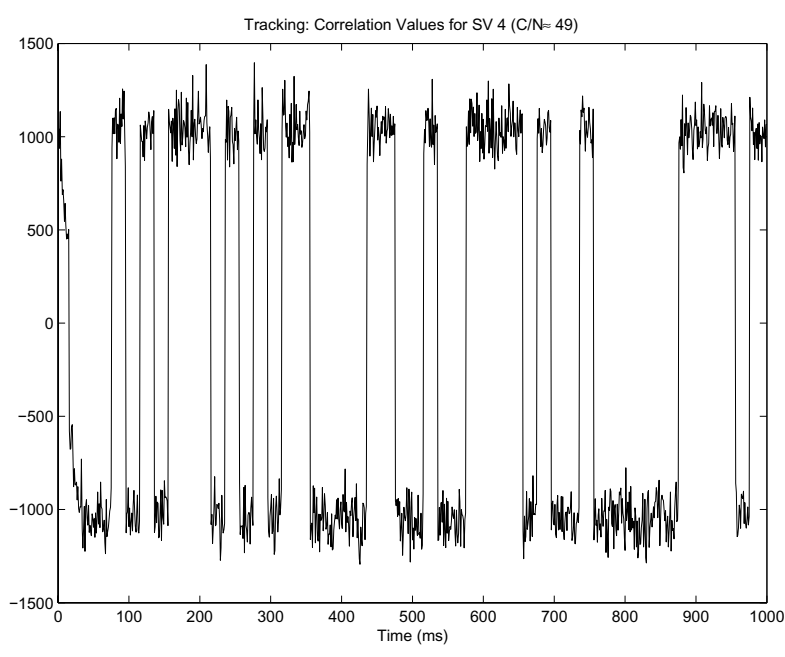

Fig. 6. Tracking results from conventional tracking phaselocked loop (Satellite SV4)

\section{REFERENCES}

[1] Dennis M Akos, A Software Radio Approach to Global Navigation Satellite System Receiver Design, $\mathrm{Ph} . \mathrm{D}$. thesis, Ohio University, Athens, Ohio, USA, August 1997.

[2] James Bao-Yen Tsui, Fundamentals of Global Positioning System Receivers - A Software Approach, A John Wiley \& Sons, Inc. Publication, 2 edition, 2005.

[3] Thomas Pany, Sung Wook Moon, Markus Irsigler, Bernd Eissfeller, and Karl Fürlinger, "Performance assessment of an under sampling SWC receiver for simulated high-bandwidth GPS/Galileo signals and real signals," in Proceedings of $16^{\text {th }}$ International Technical Meeting of the Satellite Division of The Institute of Navigation, Portland, Oregon, USA, September 9-12 2003, pp. 103-116.

[4] Falin Wu, Nobuaki Kubo, Akio Yasuda, and Harumasa Hojo, "Development of a prototyping platform for software GPS receiver," The Journal of Japan Institute of Navigation, vol. 111, pp. 193-200, September 2004, ISSN 0388-7405.

[5] Accord, GPS Signal Tap User's Guide, Accord Software \& System Private Limited, Bangalore, India, September 2003.

[6] Fredrik Johansson, Rahman Mollaei, Jonas Thor, and Jörgen Uusitalo, "GPS satellite signal acquisition and tracking," Undergraduate projects 1998, Division of Signal Processing, Luleå University of Technology, S97187 Luleå, Sweden, August 211998.

[7] Pratap Misra and Per Enge, GLOBAL POSITIONING SYSTEM, Signals, Measurements, and Performance, Ganga-Jamuna Press, 2001. 